\title{
Circular strand displacement polymerization reaction: a promising technique?
}

\begin{abstract}
"This nucleic acid isothermal amplification technology ... should be used to expand the field of point-of-care diagnostic applications making them more accessible and affordable for the masses without the need for complicated devices and highly qualified personnel."
\end{abstract}

\begin{abstract}
Keywords: circular strand displacement polymerization reaction $\approx$ colorimetric inspection hairpin probe m isothermal amplification $=$ point-of-care diagnosis
\end{abstract}

As a leading method in detecting trace amount of nucleic acids, PCR has acted as an outstanding method for target amplification that offers high specificity and sensitivity. However, the need for a thermocycling apparatus has hindered its application in point-of-care (POC) diagnosis. Various nucleic acid isothermal amplification techniques based on new discoveries in DNA/RNA synthesis and auxiliary proteins or aptamers have demonstrated the possibility of DNA amplification in vitro, thereby eliminating the need for complex devices [1].

\section{A new isothermal amplification technology}

Circular strand displacement polymerase reaction (CSDPR) is a new member of the family of isothermal amplification technologies, first introduced in 2009 by Guo et al. [2]. The designed reaction system initiates with a hairpin probe opening its stem segment, induced by a hybridization event between hairpin and target DNA. Next, a strand displacement polymerization reaction takes place in the presence of a primer and DNA polymerase, then the target DNA is displaced and triggers a new hybridization in a series of cascading reactions. This strategy has the advantage of using a hairpin probe as a template, as well as a fluorescence signal carrier to provide a simple detection method. CSDPR is a valuable tool in the detection of biological samples with single-stranded genomic DNA. Researchers have attached importance to this technology and developed various strategies and biochemical sensors to expand the application and scope of CSDPR. For example, the recently developed CSDPR technologies can not only detect single-stranded genomic DNA, but also small molecules and proteins with the aid of aptamers [3-5]. Notwithstanding these advances, further studies are still needed before we know for certain whether it is a promising technique as several weaknesses still exist in the technology (as discussed below), and the specificity, sensitivity and operability of CSDPR need to be improved.

\section{Specificity \& sensitivity}

The method's ability to discriminate base mismatches is directly related to the number of bases in the hairpin structure. The hairpin probe with a longer stem can improve mismatch discrimination ability, yet at the same time it decreases the hybridization rate and detection efficiency due to the larger activation barrier [6]. Therefore, careful design of the hairpin probe sequence is needed in order to decrease the nonspecific opening of the stemloop structure. Meanwhile, in order to solve the specificity problem associated with a single hairpin probe, it is feasible to design multiple specific hairpin probes for a target with parallel processing. For the detection of a single nucleotide polymorphism, the combination of using high-fidelity T4 DNA ligase with CSDPR has shown good potential in personalized medicine; for example in the detection of cancerrelated mutations and drug-resistant mutations of infectious agents [7].

Despite the innovative design in which each primer extension requires a smaller time interval than PCR, the original CSDPR is still a linear amplification method and the amplification efficiency is not equivalent to an exponential amplification. Some researchers have tried to introduce nicking endonuclease with two molecular switches operating in series in order to drive an exponential amplification [8].

\section{Dou Wang}

Guangzhou Institutes of Biomedicine \& Health, Chinese Academy of Sciences, Guangzhou 510530, China

Chenchen Ge

Guangzhou Institutes of Biomedicine \& Health, Chinese Academy of Sciences, Guangzhou 510530, China

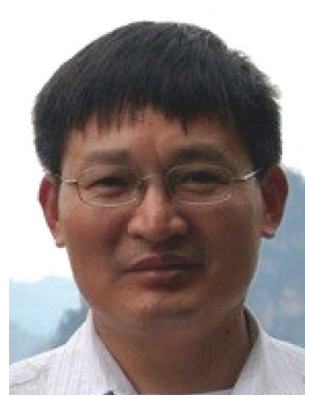

\section{Lingwen Zeng}

Author for correspondence: Guangzhou Institutes of Biomedicine \& Health, Chinese Academy of Sciences, Guangzhou 510530 , China

Tel.: +862032015312 Fax: +862032015245 Izeng8@|26.com 
It enables CSDPR to hold a higher sensibility, thereby facilitating the detection of trace amounts of DNA and narrowing the window period before the pathogenic microorganisms can be detected.

\section{How to achieve colorimetric inspection?}

Most improved CSDPR techniques require costly fluorescence measurement systems or electrochemical transducer elements. Some modifications are required to achieve colorimetric inspection, such as the loop-mediated isothermal amplification producing large amounts of white precipitate. The extensively studied G-quadruplex DNAzyme would be a good candidate. Reverse complement of the G-quadruplex sequence can be added into the hairpin probe to catalyze a color change for a signal readout, as well as reducing the need for expensive labeling procedures [WANG D \& ZENG L, Unpublished Data]. This homogeneous assay includes an amplification element, a detection transducer and a dye of acceptable specificity, as well as a format that eliminates the need for opening tube lids to avoid post-amplification manipulation and contamination. Lateral flow biosensor is another portable format to realize simple and visual readout. The use of CSDPR, coupled with a nicking endonuclease-assisted lateral flow biosensor, can lead to a DNA-detection system that operates without any qualified personnel or instruments [9]. However, any CSDPR-based detection format should not reduce the specificity and should give unequivocal results.

\section{Sample preparation is a bottleneck}

CSDPR technology also did not solve a key problem seen in other nucleic acid amplification methods. For example, sample preparation is a bottleneck, especially for trace DNA analysis, it is a cumbersome process involving specimen collection, DNA extraction and, for the case of double-stranded DNA, the need for heat denaturation to obtain single-stranded DNA for hybridization with the hairpin probe. In central laboratories, sample preparation is typically automated on large instruments. For POC nucleic acid tests, sample preparation is manually performed and requires an integrated design for all sample pretreatment steps in order to decrease time expenditure. Thus, it is advisable to synthesize all functional constituents on a disposable credit-card-sized microfluidic device. The device will reduce the consumption of samples and reagents, as well as reduce the operation complexity and the length of assay time.

\section{Still holds great promise}

In conclusion, this article briefly reviews the emergence and development of CSDPR as a promising new technology, but with some challenges and weaknesses that still need to be overcome. While this new emerging technology has some soft spots, the delicately designed hairpin structure still makes it hold great promise for biochemical assays. This technology will inspire the development of new techniques and instrumentation, such as the development of diagnostic devices for the detection of proteins, nucleic acids and toxins for biomedical applications. Future work should look at the application of this technology with an emphasis on the development of more convenient devices performed in a single tube or lateral flow biosensor without the need for complicated operations, combination of sample preparation, amplification and detection technologies that might facilitate fully integrated, low-cost POC nucleic acid test.

\section{" \\ "While this new emerging technology [circular strand displacement polymerase reaction] has some soft spots, the delicately designed hairpin structure still makes it hold great promise for biochemical assays."}

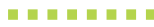

This nucleic acid isothermal amplification technology should not only act as a vehicle to compete with the widely used non-isothermal biochemical sensors or to expand the state-of-art DNA nanotechnologies, but more importantly, should be used to expand the field of POC diagnostic applications making them more accessible and affordable for the masses without the need for complicated devices and highly qualified personnel.

\section{Financial \& competing interests disclosure}

The authors have no relevant affiliations or financial involvement with any organization or entity with a financial interest in or financial conflict with the subject matter or materials discussed in the manuscript. This includes employment, consultancies, honoraria, stock ownership or options, expert testimony, grants or patents received or pending, or royalties.

No writing assistance was utilized in the production of this manuscript. 


\section{References}

1 Gill P, Ghaemi A. Nucleic acid isothermal amplification technologies: a review.

Nucleosides Nucleotides Nucleic Acids 27(3), 224-243 (2008).

2 Guo Q, Yang X, Wang K et al. Sensitive fluorescence detection of nucleic acids based on isothermal circular strand-displacement polymerization reaction. Nucleic Acids Res. 37(3), e20 (2009).

3 Ding C, Li X, Ge Y, Zhang S. Fluorescence detection of telomerase activity in cancer cells based on isothermal circular stranddisplacement polymerization reaction. Anal. Chem. 82(7), 2850-2855 (2010).

4 Huang J, Chen Y, Yang L et al. Amplified detection of cocaine based on strand- displacement polymerization and fluorescence resonance energy transfer. Biosens. Bioelectron. 28(1), 450-453 (2011).

5 Lie P, Liu J, Fang Z, Dun B, Zeng L. A lateral flow biosensor for detection of nucleic acids with high sensitivity and selectivity. Chem. Commun. 48(2), 236-238 (2012).

6 Kolpashchikov D. Binary probes for nucleic acid analysis. Chem. Rev. 110(8), 4709-23 (2010).

7 Xiao Z, Lie P, Fang Z et al. A lateral flow biosensor for detection of single nucleotide polymorphism by circular strand displacement reaction. Chem. Commun. 48(68), 8547-8549 (2012).
8 Connolly AR, Trau M. Isothermal detection of DNA by beacon-assisted detection amplification. Angew. Chem. 49(15), 2720-2723 (2010).

9 Liu J, Chen L, Lie P, Dun B, Zeng L. A universal biosensor for multiplex DNA detection based on hairpin probe assisted cascade signal amplification. Chem. Commun. 49(45), 5165-5167 (2013). 